"This accepted author manuscript is copyrighted and published by Elsevier. It is posted here by agreement between Elsevier and MTA. The definitive version of the text was subsequently published in [Hormones and Behavior, 69, March 2015, doi:10.1016/j.yhbeh.2014.12.004]. Available under license CC-BY-NC-ND."

\title{
Oxytocin induces positive expectations about ambivalent stimuli (cognitive bias) in dogs
}

Anna Kis ${ }^{\mathrm{a}, b_{*}}$, Anna Hernádi ${ }^{\mathrm{a}, \mathrm{b}}$, Orsolya Kanizsár ${ }^{\mathrm{a}, \mathrm{c}}$, Márta Gácsi ${ }^{\mathrm{d}}$, József Topál ${ }^{\mathrm{a}}$

${ }^{a}$ Research Centre for Natural Sciences, Institute of Cognitive Neuroscience and Psychology, Hungarian Academy of Sciences, Magyar Tudósok krt. 2. 1117 Budapest, Hungary

${ }^{\mathrm{b}}$ Department of Ethology, Eötvös University, Pázmány Péter stny. 1/c 1117 Budapest, Hungary

${ }^{\mathrm{c}}$ Department of Comparative Biomedicine and Food Science, University of Padova, Legnaro, PD, Italy

${ }^{\mathrm{d}}$ MTA-ELTE Comparative Ethology Research Group, Pázmány Péter stny. 1/c 1117 Budapest, Hungary

*vargane.kis.anna@ttk.mta.hu 
1 Abstract

2 Expectancy bias towards positive outcomes is a potential key to subjective well-being, and

3 has been widely investigated in different species. Here we test whether oxytocin, suggested to

4 play a role in human optimism and emotional processing, influences how dogs judge

5 ambivalent situations (in a cognitive bias paradigm). Subjects first learned in a location

6 discrimination task that a bowl either contained food (at the 'positive' location) or was empty

7 (at the 'negative' location). Then, after receiving oxytocin or placebo nasal spray, they were

8 presented with the bowl located halfway between the positive and negative positions in

9 communicative or non-communicative contexts $(\mathrm{N}=4 \times 16)$. A Positive Expectancy Score was

10 calculated for each subject using the latency to approach this ambivalent location. Compared

11 to placebo groups, subjects that received oxytocin pretreatment showed a positive expectation

12 bias in both contexts, and this effect was more pronounced in the communicative context. Our

13 study provides the first evidence for the impact of oxytocin on dogs' judgement bias and also

14 shows that the social-communicative nature of the task situation modulates the effect of 15 oxytocin.

16 Keywords: Expectation bias; Oxytocin; Dog; Canis familiaris

17

18

19

\section{Highlights}

After oxytocin administration dogs form more positive expectations about ambivalent stimuli The effect of oxytocin is enhanced by the social-communicative context of the task Dogs parallel humans in that their oxytocin system is related to expectation bias

\section{Introduction}

An increasing body of evidence supports the notion that dogs and humans (infants), in spite of their phylogenetic distance, often show comparable socio-cognitive functioning at the 
behavioural level (c.f. Senju and Csibra, 2008; Téglás et al., 2012). It has also been shown that some degree of comparability exists between dogs and humans in 'dispositional optimism', a characteristic personality feature of humans which is often conceptualized as positive expectation bias. For example, tendency to form negative ('pessimistic') judgements is associated with increased level of depressive symptoms in humans (Strunk et al., 2006) and separation related behaviour problems in dogs (Mendl et al., 2010).

Another line of recent research has provided an increasingly coherent picture of neurohormonal regulatory mechanisms of social life, suggesting that oxytocin is specifically involved in the regulation of human (and non-human) social cognition (Yamasue et al., 2012). Recent findings suggest an association between oxytocin and self-assessed psychological well-being in humans (William et al., 2011). Optimism has also long been investigated due to its role in human health and well-being (Scheier and Carver, 1992) as expectancy biases are known to be influenced both positively and negatively by people's current mood (Carver et al., 2010). Furthermore, recent research has linked such psychological resources to the oxytocin system (Saphire-Bernstein et al., 2011), although the results are still controversial (Cornelis et al., 2012).

A common way of investigating such questions in humans is by intranasally administering oxytocin (Heinrichs et al., 2009; Van IJzendoorn and Bakermans-Kranenburg, 2012) as there is a tacit assumption in the literature that this method enables direct access of the peptide to the central nervous system. However, there is no evidence yet suggesting that in dogs intranasal oxytocin administration would induce similar physiological changes as in humans.

In the current study we combine these lines of research and investigate - after validation of the physiological effects of intranasal oxytocin administration in dogs - the effects of oxytocin on positive expectations in a cognitive bias paradigm. This paradigm quantifies how subjects react (e.g. in terms of approach latency) to an ambivalent stimulus as compared to the 
51 interval determined by positive-negative stimuli (e.g. Gygax, 2014). As previous research

52 (e.g. Topál et al., 2009) has shown that the social-communicative nature of the task (whether the human experimenter addresses the subjects and makes eye-contact with them) can greatly influence dogs' performance, we decided to test the effect of oxytocin in both communicative and non-communicative test contexts.

\section{Ethical statement}

Research was done in accordance with the Hungarian regulations on animal experimentation and the Guidelines for the use of animals in research described by the Association for the Study Animal Behaviour (ASAB). Ethical approval was obtained from the National Animal Experimentation Ethics Committee (Ref No. XIV-I-001/531-4-2012).

\section{Physiological validation of intranasal oxytocin administration in dogs}

\section{Subjects and methods}

ECG recordings were conducted on ten pet dogs ( $>1$ year; 3 males and 7 females with a mean age \pm SD of $4.33 \pm 2.69$ years) following 12 IU oxytocin and placebo administration in a withinsubject design. ECG recordings were conducted in the Department of Ethology, ELTE, Budapest. The testing room was equipped with office furniture and a mattress on the floor for the dog and its owner. During a 40 minute waiting period (that is presumed to be necessary for the central oxytocin levels to reach a plateau - Born et al., 2002) dogs spent the first 25 minutes with an on-leash walk at the University Campus (avoiding any contact with other dogs or humans) during which the experimenter ensured that the owner did not make any social contact with the dog either (e.g. did not pet it, did not talk to it) and kept the length as well as the speed of the walk as standard as possible. Dogs spent the remaining 15 minutes resting in a quiet room with their passive owners present. While we made every possible 
effort to keep the circumstances of the period before the ECG measurement as standard as possible, body posture of the dog was not fully controlled by the owner/experimenter in order to avoid stress inherent to external restrain. Evidently, this procedure caused slight variations in the subjects' behaviour during the waiting period and this might have caused some noise in our data. However we expected the effect of oxytocin to be strong enough to manifest even under these semi-natural conditions. When the 40 minutes waiting period elapsed a 5-10 minutes on-leash exploration and familiarization followed in the ECG measurement room, after which the owner took a seat on the mattress and assisted the experimenter throughout the process of fixing two surface attached electrodes onto the dog's chest (second rib on both the left and right side). Gold-coated $\mathrm{Ag} \mid \mathrm{AgCl}$ electrodes fixed with EC2 Grass Electrode Cream (Grass Technologies, USA) were used for the recordings. The electrode placement was followed by 4 minutes quiet resting, and then by a 1 minute long recording. During this last five minutes every dog was in lying position because previous research has shown that body posture has a significant effect on dogs' heart rate (Maros et al., 2008). The length of the ECG measurement was based on previous dog heart rate studies (Gácsi et al., 2013; Maros et al., 2008). Signals were collected, prefiltered, amplified and digitized at a sampling rate of 249 Hz/channel by using the 30 channel Flat Style SLEEP La Mont Headbox with implemented second order filters at $0.5 \mathrm{~Hz}$ (high pass) and $70 \mathrm{~Hz}$ (low pass) as well as the HBX32-SLP 32 channel preamplifier (La Mont Medical Inc., USA). R peaks were manually detected, and RR intervals were measured using the Fercio program (C Ferenc Gombos 2012). Heart rate (HR; 1/min) was derived from RR interval averages (60/meanRR), and heart rate variability (HRV; sec) was calculated as the standard deviation of RR intervals (see e.g. Gácsi et al. 2013 for similar measures).

\section{Results}


101 In spite of the considerable individual variation in the effect of oxytocin on HR and HRV

102 (Figure 1), at the group level oxytocin significantly decreased HR $\left(\mathrm{t}_{(9)}=2.810, \mathrm{p}=0.020\right.$, 103 Cohen's $d$ : 0.944) and increased HRV ( $\mathrm{t}_{(9)}=4.472, \mathrm{p}=0.002$, Cohen's $\left.d: 1.400\right)$. These results

104 are consistent with those of previous studies on humans (Gutkowska and Jankowski, 2008;

105 Kemp et al., 2012; Kis et al., 2013; Light et al., 2005) and thus indicate that intranasal

106 administration of oxytocin can be a valid approach to study its effects in dogs. These results

107 do not provide information on the cellular mechanisms nor prove that intranasal

108 administration of oxytocin causes an increase (exclusively) in the central nervous system in

109 dogs, as peripheral increase in oxytocin levels might also lead to changes in heart rate and

110 heart rate variability due the presence of oxytocin receptors in the cardiac tissue (Jankowski et 111 al., 2004).

113 The effect of oxytocin on cognitive bias

114 Subjects

115 Sixty-four pet dogs (>1 year; 28 males, 36 females; 23 neutered; mean age \pm SD: 4.44 \pm 2.67 116 years) from various breeds (22 mongrels and 42 pure breeds from 20 different breeds) were 117 tested (21 of small ( $\leq 9 \mathrm{~kg}), 33$ of medium $(10-25 \mathrm{~kg})$ and 10 of large (>25 kg) size based on 118 average standard weight, http://www.akc.org/ in case of pure breed dogs or based on the 119 inspection of the videos in case of mixed breed dogs). In order to be eligible for the test dogs 120 needed to be motivated by dry food according to their owners. Subjects were randomly 121 assigned into four experimental conditions: receiving oxytocin (OT) or placebo (PL) pre122 treatment and participating in the test in either communicative (Com) or non-communicative 123 (NCom) context $(\mathrm{N}=16$ in each). The four groups did not differ in mean age (ANOVA, $124 \mathrm{~F}=0.457, \mathrm{p}=0.714)$, sex ratio $\left(\mathrm{Chi}^{2}\right.$ test, $\left.\chi^{2}=3.089, \mathrm{p}=0.386\right)$, neutered status $\left(\chi^{2}=3.052\right.$, $125 \mathrm{p}=0.384)$, pure/mixed breed $\left(\chi^{2}=1.140, \mathrm{p}=0.767\right)$, or size $\left(\chi^{2}=0.746, \mathrm{p}=0.993\right)$. Owners were 
126 blind to the aims of the study and details of the experimental procedure as well as to the

127 pretreatment their dogs received.

\section{Procedure}

130 The summary of the procedure is shown in Figure 2, the video-protocol can be accessed

131 through the following link: http://www.cmdbase.org/web/guest/play/-/videoplayer/249. The

132 whole procedure was carried out on the same day and took approximately 50-60 minutes

133 (training phase: 5-15 minutes, pretreatment: 1-2 minutes, waiting period: 40 minutes,

134 retraining: 3 minutes, test phase: 1 minute) that is presumed to be within the time interval 135 when intranasally administered oxytocin exerts its central effects (see e.g. Ditzen et al., 2009;

136 Heinrichs et al., 2003).

$138 \quad \underline{\text { Training }}$

139 The training phase was identical for all subjects and was based on the procedure developed by

140 (Mendl et al., 2010). The dog was held by its collar by the owner at a predetermined starting

141 point, at a $3 \mathrm{~m}$ distance from the two possible hiding locations placed on the left and right side

142 of the room $2 \mathrm{~m}$ apart from each other. The experimenter positioned herself facing the dog,

143 established eye-contact with it, and addressed it (dog's name + "Look!"). Then she placed the

144 food bowl to either of the two locations (i.e. positive $-\mathrm{P}$ and negative $-\mathrm{N}$ ) in a fixed semi-

145 random order (PPNPNN, repeated until criterion - see below), so that at the positive side the

146 bowl always contained a food reward while at the negative side it was always empty. Dogs

147 and their owners had no visual access to the content of the bowl except for the first trial (this

148 was a positive trial for all subjects) when the experimenter, before hiding the food, showed it

149 up in order to motivate the dog to search. The positive and negative side (left/right) was

150 counterbalanced across subjects. The owner was instructed to release the dog at the moment, 
151 when the food bowl touched the ground. If the dog did not start moving when released, the

152 owner was allowed to encourage it with short utterances (e.g. "Go!", "It's yours") or by

153 gently touching it. Apart from this no other forms of communication were allowed. The dog

154 was allowed to approach the food bowl in every trial, while the experimenter was looking

155 straight ahead without maintaining eye contact with the dog. The latency of approach (i.e. the

156 time elapsed between the moment when the food bowl touched the ground and the dog

157 reached the line of the food bowl) was noted in every trial. Although there was some variation

158 in owners' reaction times releasing their dogs, we decided to use the moment when the food

159 bowl touched the ground as a starting point because this could always be coded reliably, while

160 owners did not always release their dogs with an easily visible movement. In order to exclude

161 the possibility that the owners' reaction times (e.g. the time elapsed between the bowl

162 touching the ground and the owner releasing the dog) systematically influenced our latency

163 data we coded owner reaction times in case of 32 subjects (50\% of the total sample) and

164 found no difference in the test trials among the four treatment groups $(\mathrm{OT} / \mathrm{PL} \times \mathrm{Com} / \mathrm{NCom}$;

165 ANOVA, $\mathrm{F}=1.707, \mathrm{p}=0.188)$ nor among the positive/negative/ambivalent trials $(\mathrm{F}=0.327$,

$166 \mathrm{p}=0.722$ ). Successive trials were presented with no breaks in between. Dogs were deemed to

167 have learnt an association between bowl location and food reward when for the preceding five

168 positive trials and the preceding five negative trials the longest latency to reach the positive

169 location was shorter than any of the latencies to reach the negative location (Wilcoxon Test,

$170 \mathrm{p}=0.025)$. This took on average $23 \pm 6(\mathrm{mean} \pm \mathrm{SD})$ trials, with a minimum of 12 and a

171 maximum of 36 trials.

172

173 Pretreatment

174 After having reached this learning criterion half of the subjects received a single intranasal

175 dose of 12 IU oxytocin (Syntocinon-Spray, Novartis; nasal spray with a nebulizer) (OT, 
$176 \mathrm{~N}=32$ ), the other half received placebo, isotonic natriumchlorid $0.9 \%$ solution (PL, N=32).

177 The 12 IU dose was chosen to be half of the 24 IU commonly used in human studies (e.g.

178 Lischke et al., 2012; Perry et al., 2010), and the same dose was administered to all subjects

179 irrespective of their body weight (which is also the common practice in human studies). Then,

180 a 40-minute waiting period followed, divided into a 25 minutes on-leash walk and a 15

181 minutes quiet resting in the exact same way as described in the ECG study.

182

183 Re-training

184 After the waiting period had elapsed dogs participated in a 9-trial re-training phase that, in

185 case of the communicative context (half of the subjects, 16 OT and 16 PL), was identical to

186 the training trials, while in the non-communicative context (other half of the subjects, 16 OT

187 and 16 PL) the experimenter acted from behind a curtain, and slid the food bowl under the

188 curtain into position, without providing any communicative cues. (This re-training phase was

189 necessary because our pilot data showed that dogs' latency to reach the food bowl did not

190 differ between the positive and negative sides after a 40 minutes delay that followed the

191 training.)

192

193 Test phase

194 The test phase consisted of three trials: a negative $(\mathrm{N})$, a positive $(\mathrm{P})$ and an ambivalent $(\mathrm{A}$;

195 during which the baited bowl was placed halfway between the positive and negative locations

196 (17 cm behind the line connecting the two locations, at a $3 \mathrm{~m}$ distance from the dog) trial. The

197 trials were presented in fixed (N, P, A) order administered in the same Com or NCom context

198 (half of the subjects participating in each context) as described for the re-training. The

199 training, re-training and test phases were videotaped and the latencies to approach the food

200 bowl were coded with a frame-by-frame analysis using Solomon Coder 
(http://solomoncoder.com/) blind to OT/PL treatment of the subjects. Inter-rater reliability was calculated for both the start and the end point of the latency based on double coding of 13 recordings ( $20 \%$ of the total sample) and resulted in an almost perfect agreement between the two raters (start: $\kappa=1.00$, end: $\kappa=0.83$ ).

Although one could argue that dogs in this situation can possibly smell whether there is food in the bowl, previous research (e.g. Lakatos et al., 2011) indicates that in similar setups dogs are not able to choose the baited cup based on odour cues alone. (This is further supported by the fact that our subjects did not differentiate in their latency to reach the positive versus negative location (paired samples t-test, $\mathrm{t}_{(65)}=0.553, \mathrm{p}=0.582$ ) in their first training trials.)

\section{Data analysis}

Training phase. Mean latency to approach the positive and negative locations was calculated for each subject based on the last five positive and the last five negative trials. A Generalized Estimating Equation (GEE) model was used to confirm the effect of location (positive vs. negative; within subject factor) on the latency to approach the bowl and to test the possible differences among the four conditions (between subject factor).

Re-training phase. For each subject the latency for the first positive and the first negative as well as the last positive and the last negative trial was entered in a GEE model with the following factors: positive vs. negative trial (within subject factor), first vs. last trial (within subject factor), Com vs. NCom context (between subject factor), OT vs. PL pretreatment (between subject factor).

Test phase. A GEE was used to test the differences between the latency to approach the positive vs. negative vs. ambivalent location (within subject factor) and the effect of test context (Com vs. NCom; between subject factor) as well as the effect of pretreatment (OT vs. PL, between subject factor). Moreover, in order to assess subjects' judgement bias in the 
ambivalent trials and to control for the high individual variation in running speed (which presumably causes a greater variation than the treatment itself), a Positive Expectancy Score (PES) was calculated for each subject using the latency to approach the negative, positive and ambivalent locations according to the following formula: PES $=100-\mathrm{CBS}$, where

$$
\mathrm{CBS}=\frac{(\text { latency toreach ambivalentlocation- latency toreach positivelocation }) * 100}{\text { latency toreach negativelocation- latency toreach positivelocation }}
$$

Note, that CBS is the adjusted cognitive bias score previously developed by Mendl et al. (2010).

Higher PES values thus indicate a more positive expectation bias (the latency for the ambivalent location is more similar to the latency for the positive than for the negative location). In cases when the latency for the ambivalent location is in-between the latency to the negative and to the positive location (with a higher negative latency) the value of the PES falls within the 0-100 interval.

A General Linear Model (GLM) was used to test the effect of test context (Com vs. NCom; between subject factor) as well as the effect of pretreatment (OT vs. PL; between subject factor) on PES. Planned pairwise comparisons (independent samples t-tests) were carried out to assess the effect of OT vs. PL pretreatement in both the Com and NCom contexts; as well as to assess the effects of Com vs. NCom test contexts for both OT and PL pretreated dogs. The effect size (Cohen's D) was calculated using the www.cognitiveflexibility.org/effectsize/ webpage. The effect of independent variables (sex, neutered status, age, pure / mix breed, size) and the interaction within these factors and with the four groups (OT/PL pretreatment $\times$ Com/NCom context) was tested with a GLM. All statistical tests were two-tailed with an alpha value of $\alpha=0.05$.

\section{Results}


The GEE analysis revealed that by the end of the training phase there was a consistent

251 difference in the latency to approach the positive versus negative location (with a shorter 252 latency for the positive location; $\chi^{2}=55.215$, $\mathrm{p}<0.001$ ) while the four conditions did not differ 253 from each other $\left(\chi^{2}=3.827, \mathrm{p}=0.281\right)$ and there was no significant condition $\times$ location 254 interaction $\left(\chi^{2}=3.123, \mathrm{p}=0.373\right)$.

255 Raw latency data for the different conditions of the re-training and test phases are shown in 256 Table 1. In the re-training phase subjects' latencies were higher in the NCom than in the Com context $\left(\chi^{2}=13.089, \mathrm{p}<0.001\right)$, and a significant positive/negative location $\times$ first/last trial interaction $\left(\chi^{2}=16.361, \quad \mathrm{p}<0.001\right)$ indicated that subjects differentiated between positive/negative locations only at the end of the re-training, but not at the beginning. The effect of OT/PL pretreatment $\left(\chi^{2}=0.159, p=0.690\right)$ as well as all other interactions were not significant (all $\mathrm{p}>0.05)$.

During the test phase a similar difference was found between the positive, negative and ambivalent locations (GEE, $\chi^{2}=38.353, \mathrm{p}<0.001$ ). Furthermore, dogs in the NCom context showed higher latencies $\left(\chi^{2}=15.444, \mathrm{p}<0.001\right)$ irrespective of PL/OT pretreatment $\left(\chi^{2}=0.002\right.$, $\mathrm{p}=0.963)$. However there was a significant $\mathrm{P} / \mathrm{N} / \mathrm{A}$ location $\times \mathrm{PL} / \mathrm{OT}$ pretreatment interaction $\left(\chi^{2}=8.678, p=0.013\right)$ indicating that the OT effect was specific to the ambivalent location (see also the results for the PES score) as well as a P/N/A location $\times$ Com/NCom context interaction $\left(\chi^{2}=8.721, \mathrm{p}=0.013\right)$. The PL/OT pretreatment Com/NCom context interaction did not reach significance $\left(\chi^{2}=2.732, \mathrm{p}=0.098\right)$, and neither did the three-way interaction $\left(\chi^{2}=1.780, \mathrm{p}=0.411\right)$. More importantly, dogs receiving OT pretreatment achieved a higher 271 Positive Expectancy Score (PES), than dogs receiving PL pretreatment (GLM, F=38.818, $\mathrm{p}<0.001)$ and this difference was more pronounced in the communicative context as reflected

273 in a significant pretreatment $\times$ context interaction $(F=5.434, p=0.023$, Figure 3.). There was 274 no main effect of Com/NCom contexts $(\mathrm{F}=1.952, \mathrm{p}=0.167)$. 
275 Planned pairwise comparisons confirmed these results as OT pretreated dogs achieved higher

276 PES both in the Com $\left(\mathrm{t}_{(30)}=6.118, \mathrm{p}<0.001\right.$, Cohen's $\left.d: 2.163\right)$ and in the NCom $\left(\mathrm{t}_{(30)}=2.729\right.$, $277 \mathrm{p}=0.011$, Cohen's d: 0.965) contexts. Furthermore, OT pretreated dogs achieved a higher PES 278 in the Com than in the NCom context $\left(\mathrm{t}_{(30)}=2.884, \mathrm{p}=0.007\right.$, Cohen's $\left.d: 1.020\right)$, whereas PL 279 pretreated dogs did not show a context dependent difference $\left(\mathrm{t}_{(30)}=0.612, \mathrm{p}=0.545\right.$, Cohen's $d$ : $280 \quad 0.216)$.

281 The PES was not affected by the subjects' sex $(\mathrm{F}=0.231, \mathrm{p}=0.644)$, neutered status $(\mathrm{F}=0.158$, 282 $\mathrm{p}=0.701)$, age $(\mathrm{F}=0.032, \mathrm{p}=0.862)$, breed $(\mathrm{F}=0.652, \mathrm{p}=0.443)$ or size $(\mathrm{F}=0.099, \mathrm{p}=0.761)$, and there was no significant interaction among these factors or with the pretreatment group (all $284 \mathrm{p}>0.05)$.

\section{Discussion}

This study presents new information in the growing debate over whether oxytocin modulates positive expectation bias in humans (Cornelis et al., 2012; Saphire-Bernstein et al., 2011) or in nonhuman animals. Our results validate the effect of intranasal oxytocin administration for dogs at the physiological level (although without uncovering the exact mechanisms) and 291 provide the first evidence suggesting that oxytocin induces positive expectations in dogs. Recent research has provided an increasingly coherent picture of the involvement of oxytocin in the regulation of human and non-human social behaviour phenomena (such as trust (Kosfeld et al., 2005) and generosity (Barraza et al., 2011) or social memory (Ferguson et al., 2002; Guastella et al., 2008)), and in our study the judgement bias in dogs about ambivalent 296 stimuli also appears to be modulated by the social-communicative nature of the task context. The differential effects of the communicative and non-communicative contexts on dogs behaviour might be due to several factors such as the presence of the experimenter providing 
human (especially in the ambivalent trial when the food bowl was placed in the middle

301 position). Interestingly, however, this effect was selectively associated with oxytocin pretreatment which may indicate an interspecific (dog-human) social-tuning effect of this neuromodulator in the dog.

304 The present findings extend our previous knowledge about the role of oxytocin in positive 305 emotions and welfare (Mitsui et al., 2011) and reveal an interesting parallel between dogs and 306 humans with regard to the connectedness between the oxytocin system and positive 307 expectation bias. Human optimism as well as the 'optimistic/pessimistic' cognitive bias in 308 animal models (Harding et al., 2004) have been linked to mental health (Scheier and Carver, 3091987,1985 ) and behavioural problems (such as separation anxiety - Mendl et al., 2010) as 310 well as to placebo sensitivity (humans: Geers et al., 2005; dogs: Sümegi et al., 2014). Our 311 results, therefore, may have potential applied and some indirect clinical relevance. We note, 312 however, that further studies should determine how other factors, such as 'baseline optimism' 313 of the subjects and/or polymorphisms in the OXTR gene, modulate the effect we have found. 314 Recent accounts in the human literature cautioned about the individual differences in the 315 effects of oxytocin on social behavior (Bartz et al., 2011), and a recent study on dogs also 316 found that the effect of a polymorphism in the OXTR gene on social behaviour is conditional 317 to a breed effect (Kis et al., 2014).

318 It is also important that previous studies (e.g. Mendl et al. 2010) found that their 'treatment' 319 had a similar effect on both the latency to reach the ambivalent location and the adjusted score 320 calculated based on latency to positive/negative/ambivalent locations. In the current study, 321 however, we found a main effect of PL/OT treatment only in case of the PES score, while in 322 the analysis of the raw latency data the effect of treatment was in interaction with other 323 factors. This seemingly contradictory finding might be explained by the fact that the subjects 324 of the present study were very heterogeneous (randomly selected from a pet dog database vs. 
dogs were from two animal re-homing centres in the Mendl et al. 2010 study) and thus their running speed varied greatly, causing a greater variation in the latency data, than the effect of the treatment. These individual variations were controlled for in the PES score that was calculated based on the latency to positive/negative/ambivalent locations and thus used an individual negative-positive scale for each dog. It can be argued that because dogs received 330 the test trials in a fixed negative, positive, ambivalent order, and the ambivalent trial was 331 always preceded by a positive trial, this might have biased the PES score towards the positive direction. However we found no such deviation from the chance level in the placebo groups. Also, even if such a bias had existed, it would have been the same for all subjects, not affecting the revealed effect of oxytocin and social-communicative task context.

335 Contrary to expectations (e.g. Herzmann et al., 2013), in the present study dogs' sex, neutered 336 status, age, breed (pure / mix breed) or size did not have an effect on dogs' positive expectancy nor was in interaction with pretreatment. The most parsimonious interpretation of these results is that as the goal of the present study was not to unravel individual differences, 339 we could not find such potential effects due to the limited sample size and the lack of 340 systematic grouping based on these features. We should also note that a further factor that 341 might contribute to individual variations in oxytocin effects in both the present and previous 342 human studies, is the fact that all subjects receive the same dose of intranasal oxytocin 343 irrespective of body weight. Due to the large within-species variation this confound might be 344 more pronounced in dogs.

345 Previous research has shown that the dog is a promising model species to study human 346 psychiatric conditions (Overall, 2000) as well as the genetic background of certain illnesses 347 (Parker et al., 2010). The present results extend these notions by showing that a similar neuro348 hormonal mechanism (the oxytocin system) might be responsible for a crucial psychological 349 resource, the positive judgement of ambivalent stimuli. Importantly, in addition to ample 
350 evidence on the role of oxytocin in regulating social behaviour in humans and rodents 351 (Donaldson and Young, 2008), this is the first evidence of the effect of intranasally 352 administered oxytocin on dog behaviour, and thus our results open up the way for further 353 research to use the dog as a model of human socio-cognitive competences (Miklósi and 354 Topál, 2013) at the neurohormonal level as well.

355

356 Acknowledgement

357 Financial support was provided by the Hungarian Scientific Research Fund (OTKA K100695) 358 and the Hungarian Academy of Sciences (MTA 01031 ). 


\section{References}

Barraza, J.A., McCullough, M.E., Ahmadi, S., Zak, P.J., 2011. Oxytocin infusion increases charitable donations regardless of monetary resources. Horm. Behav. 60, 148-151.

Bartz, J.A., Zaki, J., Bolger, N., Ochsner, K.N., 2011. Social effects of oxytocin in humans: context and person matter. Trends Cogn. Sci. 15, 301-309.

Born, J., Lange, T., Kern, W., McGregor, G.P., Bickel, U., Fehm, H.L., 2002. Sniffing neuropeptides: a transnasal approach to the human brain. Nat. Neurosci. 5, 514-516.

Carver, C.S., Scheier, M.F., Segerstrom, S.C., 2010. Optimism. Clin. Psychol. Rev. 30, 879889.

Cornelis, M.C., Glymour, M.M., Chang, S.-C., Tchetgen, E.J.T., Liang, L., Koenen, K.C., Kang, J.H., Pasquale, L.R., Rimm, E.B., Kawachi, I., Kubzansky, L.D., 2012. Oxytocin receptor (OXTR) is not associated with optimism in the Nurses' Health Study. Mol. Psychiatry 17, 1157-1159.

Ditzen, B., Schaer, M., Gabriel, B., Bodenmann, G., Ehlert, U., Heinrichs, M., 2009. Intranasal oxytocin increases positive communication and reduces cortisol levels during couple conflict. Biol. Psychiatry 65, 728-31.

Donaldson, Z.R., Young, L.J., 2008. Oxytocin, vasopressin, and the neurogenetics of sociality. Science (80-. ). 322, 900-904.

Ferguson, J.N., Young, L.J., Insel, T.R., 2002. The neuroendocrine basis of social recognition. Front. Neuroendocrinol. 23, 200-224.

Gácsi, M., Maros, K., Sernkvist, S., Faragó, T., Miklósi, Á., 2013. Human analogue safe haven effect of the owner: Behavioural and heart rate response to stressful social stimuli in dogs. PLoS One 8, e58475.

Geers, A.L., Helfer, S.G., Kosbab, K., Weiland, P.E., Landry, S.J., 2005. Reconsidering the role of personality in placebo effects: dispositional optimism, situational expectations, and the placebo response. J. Psychosom. Res. 58, 121-7.

Guastella, A.J., Mitchell, P.B., Mathews, F., 2008. Oxytocin enhances the encoding of positive social memories in humans. Biol. Psychiatry 64, 256-258.

Gutkowska, J., Jankowski, M., 2008. Oxytocin revisited: It is also a cardiovascular hormone. J. Am. Soc. Hypertens. 2, 318-325.

Gygax, L. 2014. The A to Z of statistics for testing cognitive judgement bias. Anim. Behav. 95, 59-69.

Harding, E.J., Paul, E.S., Mendl, M., 2004. Cognitive bias and affective state. Nature 427, 321. 
Heinrichs, M., Baumgartner, T., Kirschbaum, C., Ehlert, U., 2003. Social support and oxytocin interact to suppress cortisol and subjective responses to psychosocial stress. Biol. Psychiatry 54, 1389-1398.

Heinrichs, M., Dawans, B. Von, Domes, G., von Dawans, B., 2009. Oxytocin, vasopressin, and human social behavior. Front. Neuroendocrinol. 30, 548-557.

Herzmann, G., Bird, C., Freeman, M., Curran, T., 2013. Effects of oxytocin on behavioral and ERP measures of recognition memory for own-race and other-race faces in women and men. Psychoneuroendocrinology 38, 2140-2151.

Jankowski, M., Danalache, B., Wang, D., Bhat, P., Hajjar, F., Marcinkiewicz, M., Paquin, J., McCann, S.M., Gutkowska, J., 2004. Oxytocin in cardiac ontogeny. Proc. Natl. Acad. Sci. U. S. A. 101, 13074-9.

Kemp, A.H., Quintana, D.S., Kuhnert, R.-L., Griffiths, K., Hickie, I.B., Guastella, A.J., 2012. Oxytocin increases heart rate variability in humans at rest: Implications for social approach-related motivation and capacity for social engagement. PLoS One 7, e44014.

Kis, A., Bence, M., Lakatos, G., Pergel, E., Turcsán, B., Pluijmakers, J., Vas, J., Elek, Z., Brúder, I., Földi, L., Sasvári-Székely, M., Miklósi, Á., Rónai, Z., Kubinyi, E., 2014. Oxytocin receptor gene polymorphisms are associated with human directed social behavior in dogs (Canis familiaris). PLoS One 9, e83993.

Kis, A., Kemerle, K., Hernádi, A., Topál, J., 2013. Oxytocin and social pretreatment have similar effects on processing of negative emotional faces in healthy adult males. Front. Psychol. 4, 532.

Kosfeld, M., Heinrichs, M., Zak, P.J., Fischbacher, U., Fehr, E., 2005. Oxytocin increases trust in humans. Nature 435, 673-676.

Lakatos, G., Gácsi, M., Topál, J., Miklósi, Á., 2011. Comprehension and utilisation of pointing gestures and gazing in dog-human communication in relatively complex situations. Anim. Cogn. 15, 18-22.

Light, K.C., Grewen, K.M., Amico, J. a, 2005. More frequent partner hugs and higher oxytocin levels are linked to lower blood pressure and heart rate in premenopausal women. Biol. Psychol. 69, 5-21.

Lischke, A., Berger, C., Prehn, K., Heinrichs, M., Herpertz, S.C., Domes, G., 2012. Intranasal oxytocin enhances emotion recognition from dynamic facial expressions and leaves eyegaze unaffected. Psychoneuroendocrinology 37, 475-481.

Maros, K., Doka, a, Miklósi, Á., 2008. Behavioural correlation of heart rate changes in family dogs. Appl. Anim. Behav. Sci. 109, 329-341.

Mendl, M., Brooks, J., Basse, C., Burman, O., Paul, E., Blackwell, E., Casey, R., 2010. Dogs showing separation-related behaviour exhibit a "pessimistic" cognitive bias. Curr. Biol. 20, R839-R840. 
Miklósi, Á., Topál, J., 2013. What does it take to become "best friends"? Evolutionary changes in canine social competence. Trends Cogn. Sci. 17, 287-294.

Mitsui, S., Yamamoto, M., Nagasawa, M., Mogi, K., Kikusui, T., Ohtani, N., Ohta, M., 2011. Urinary oxytocin as a noninvasive biomarker of positive emotion in dogs. Horm. Behav. 60, 239-243.

Overall, K.L., 2000. Natural animal models of human psychiatric conditions: assessment of mechanism and validity. Prog. Neuro-Psychopharmacology Biol. Psychiatry 24, $727-$ 776.

Parker, H.G., Shearin, A.L., Ostrander, E. a, 2010. Man's best friend becomes biology's best in show: genome analyses in the domestic dog. Annu. Rev. Genet. 44, 309-336.

Perry, A., Bentin, S., Shalev, I., Israel, S., Uzefovsky, F., Bar-on, D., Ebstein, R.P., 2010. Intranasal oxytocin modulates EEG mu / alpha and beta rhythms during perception of biological motion. Psychoneuroendocrinology 35, 1446-53.

Saphire-Bernstein, S., Way, B.M., Kim, H.S., Sherman, D.K., Taylor, S.E., 2011. Oxytocin receptor gene (OXTR) is related to psychological resources. Proc. Natl. Acad. Sci. 108, $15118-15122$.

Scheier, M.F., Carver, C.S., 1985. Optimism, coping, and health: assessment and implications of generalized outcome expectancies. Heal. Psychol. 4, 219-247.

Scheier, M.F., Carver, C.S., 1987. Dispositional optimism and physical well-being: the influence of generalized outcome expectancies on health. J. Pers. 55, 169-210.

Scheier, M.F., Carver, C.S., 1992. Effects of optimism on psychological and physical wellbeing: Theoretical overview and empirical update. Cognit. Ther. Res. 16, 201-228.

Senju, A., Csibra, G., 2008. Gaze following in human infants depends on communicative signals. Curr. Biol. 18, 668-671.

Strunk, D.R., Lopez, H., DeRubeis, R.J., 2006. Depressive symptoms are associated with unrealistic negative predictions of future life events. Behav. Res. Ther. 44, 861-882.

Sümegi, Z., Gácsi, M., Topál, J., 2014. Conditioned placebo effect in dogs decreases separation related behaviours. Appl. Anim. Behav. Sci. 158, 90-98.

Téglás, E., Gergely, A., Kupán, K., Miklósi, Á., Topál, J., 2012. Dogs’ gaze following is tuned to human communicative signals. Curr. Biol. 22, 209-212.

Topál, J., Gergely, G., Erdőhegyi, Á., Csibra, G., Miklósi, Á., Mikló, 2009. Differential sensitivity to human communication in dogs, wolves, and human infants. Science (80-. ). 325, 1269-1272.

Van IJzendoorn, M.H., Bakermans-Kranenburg, M.J., 2012. A sniff of trust: meta-analysis of the effects of intranasal oxytocin administration on face recognition, trust to in-group, and trust to out-group. Psychoneuroendocrinology 37, 438-443. 
466 William, W., Kahloon, M., Fakhry, H., Ishak, W.W., 2011. Oxytocin role in enhancing well467 being: a literature review. J. Affect. Disord. 130, 1-9.

468 Yamasue, H., Yee, J.R., Hurlemann, R., Rilling, J.K., Chen, F.S., Meyer-Lindenberg, A., 469 Tost, H., 2012. Integrative approaches utilizing oxytocin to enhance prosocial behavior: 470 from animal and human social behavior to autistic social dysfunction. J. Neurosci. 32, $471 \quad 14109-14117$. 


\section{$474 \quad$ Figure legends}

\section{$475 \quad$ Figure 1.}

476 The effect of oxytocin on heart rate and heart rate variability in ten individual dogs
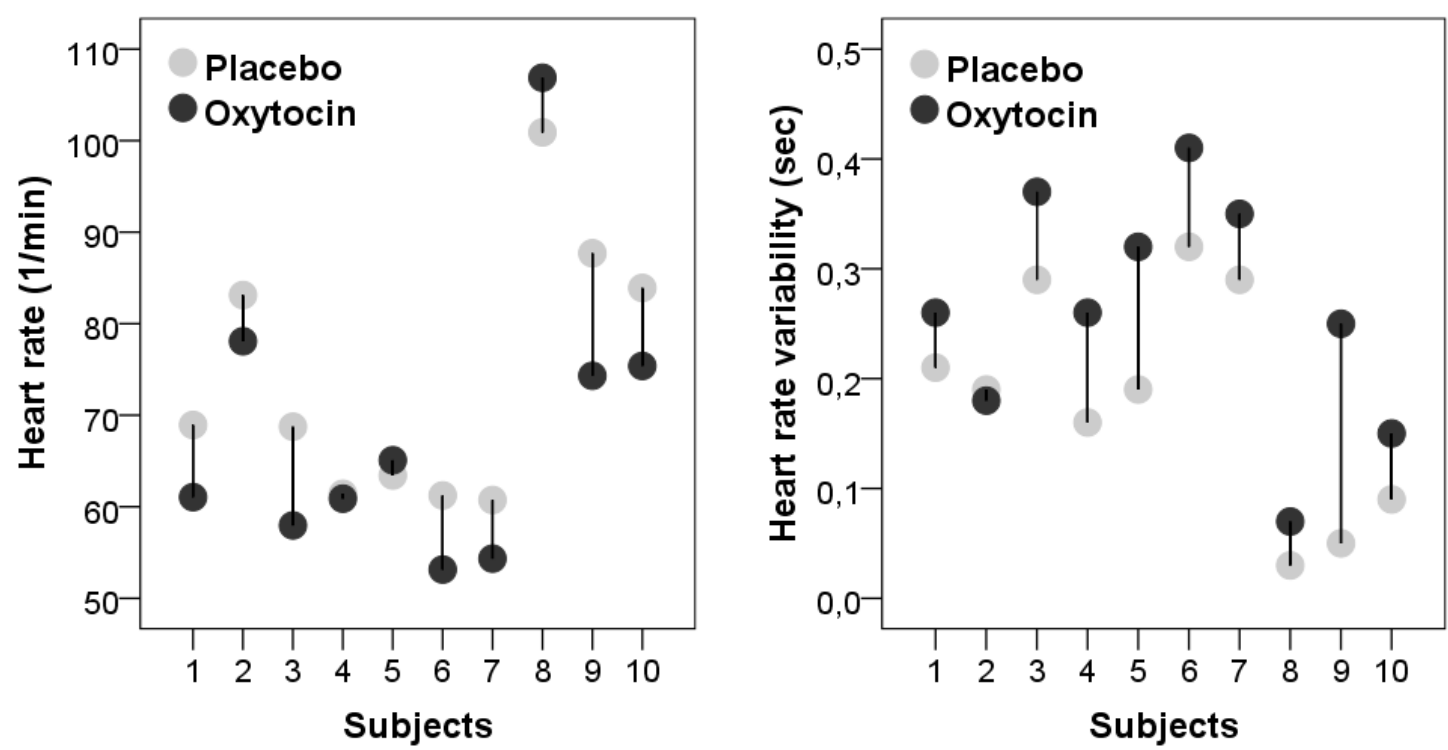


\section{Figure 2.}

479 Schematic overview of the different conditions throughout the four phases of the experiment.

480 The drawings in one row stand for the different type of trials (e.g. positive and negative) that 481 one individual subject received in that phase, while the different rows represent between 482 subject treatments (e.g. communicative or non-communicative)

1) Training Phase (Positive \& Negative trials; until criterion)
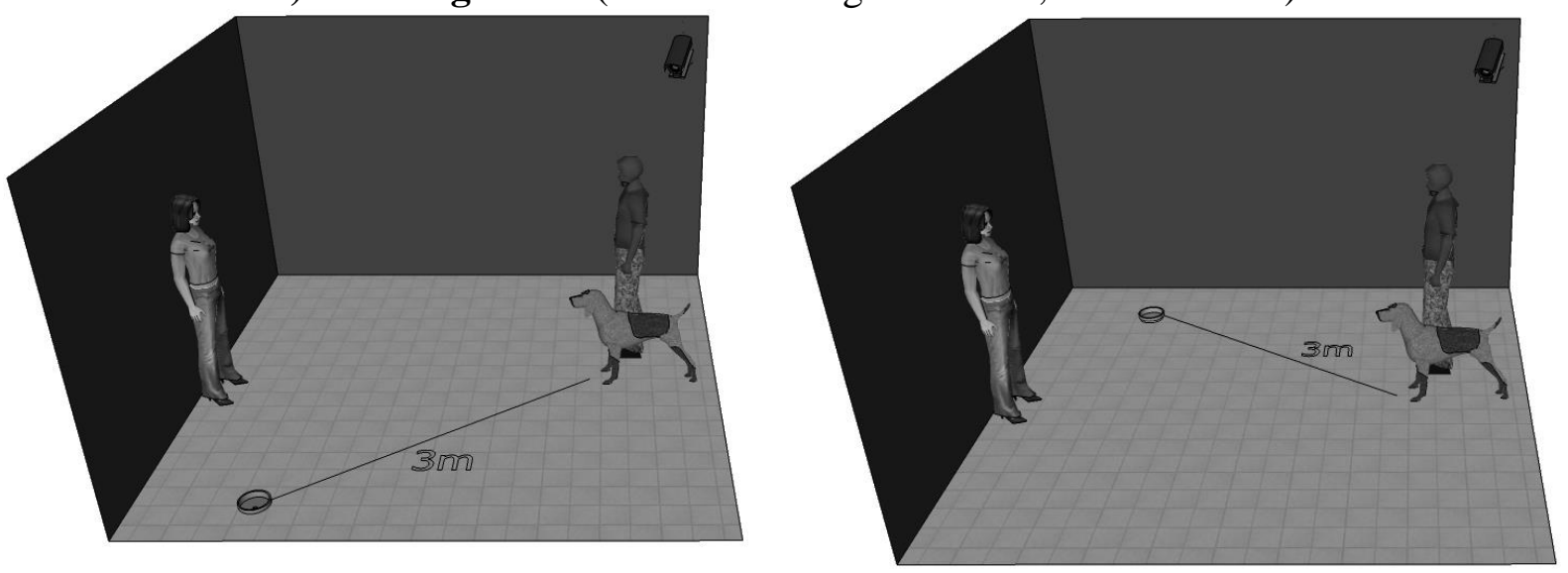

2) Pretreatment (OT or PL nasal spray +40 minutes waiting period)

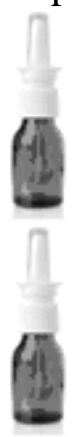

3) Re-training Phase (Positive \& Negative trials in Com or NCom context; 9 trials)
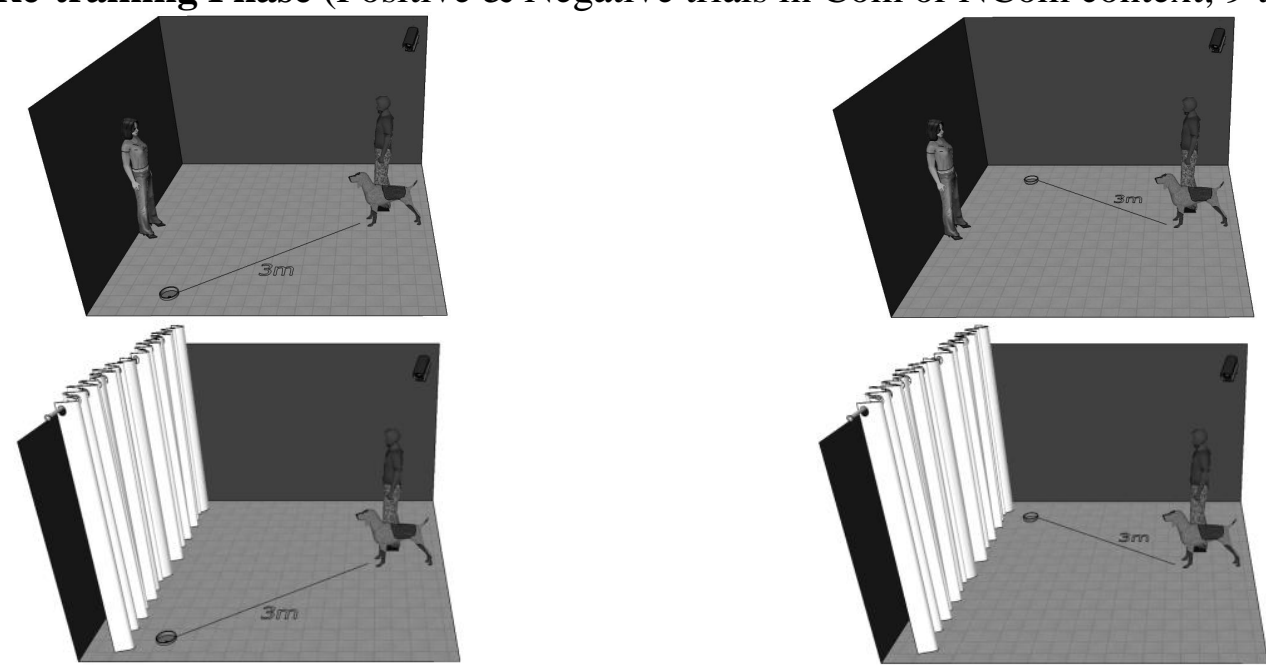

4) Test Phase (1-1-1 Negative-Positive-Ambivalent trial in Com or NCom context) 
Kis et al.
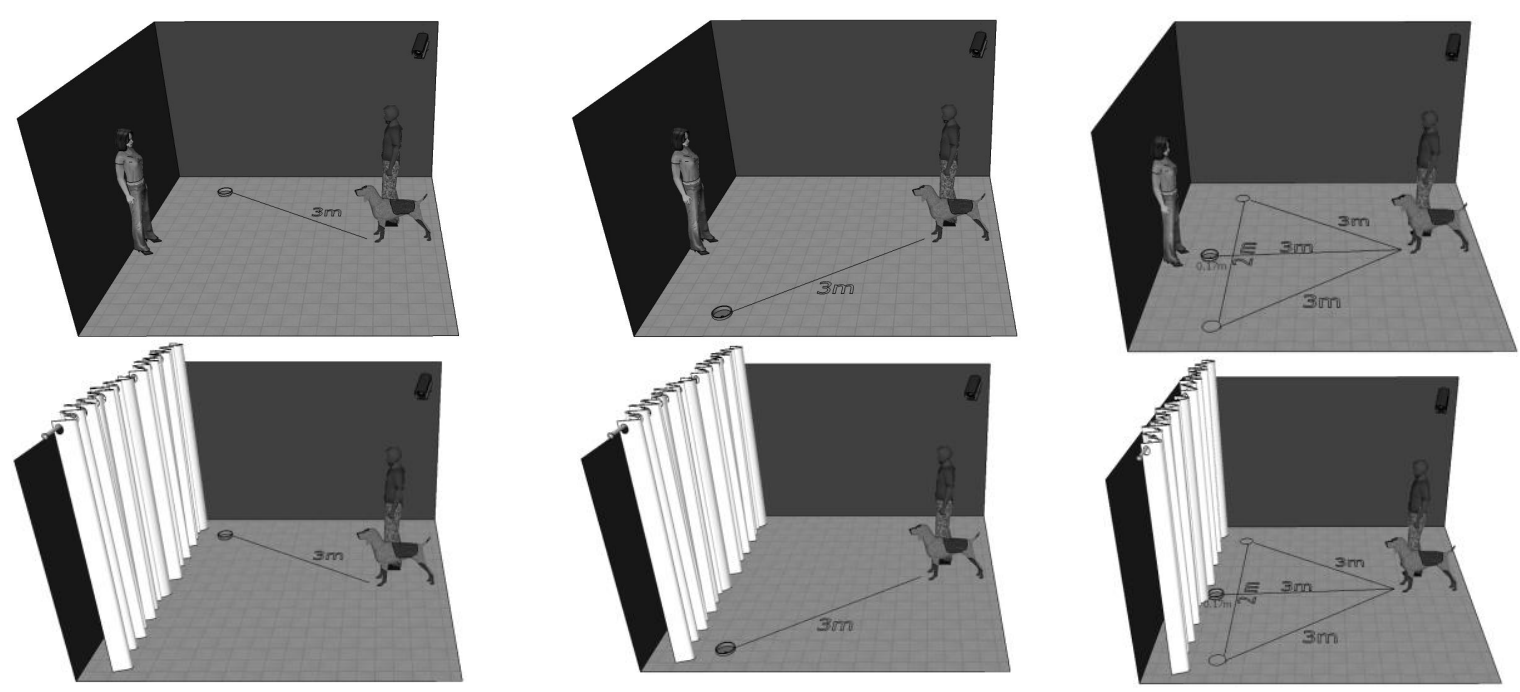


\section{$484 \quad$ Figure 3.}

485 The Positive Expectancy Scores (PES) of dogs in the non-communicative and social-

486 communicative versions of the cognitive bias task after placebo / oxytocin pretreatment

487 (mean \pm SE). A higher PES indicates a reaction to the ambivalent location that is more similar 488 to the reaction to the positive than to the negative location. *: $p<0.05$, ***: $p<0.001$

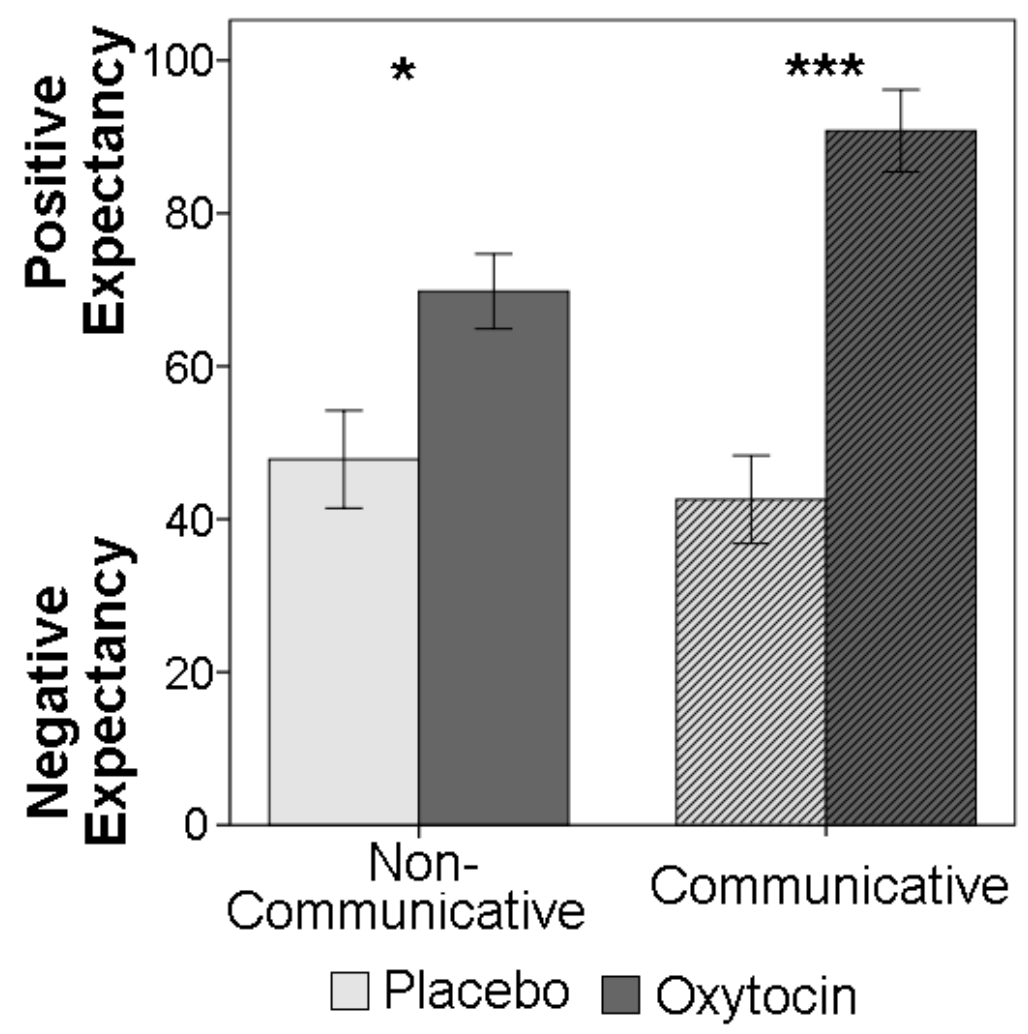

489 
491 Table 1.

492 Latency mean $\pm S D$ ( $\min -\max$ ) to reach the food bowl in the different conditions of the re-

493 training and test phases (sec). Data for the PL/OT pretreated dogs is pulled together for the re-

494 training phase as the statistical analysis showed no effect of treatment on the positive/negative

495 latencies during this phase

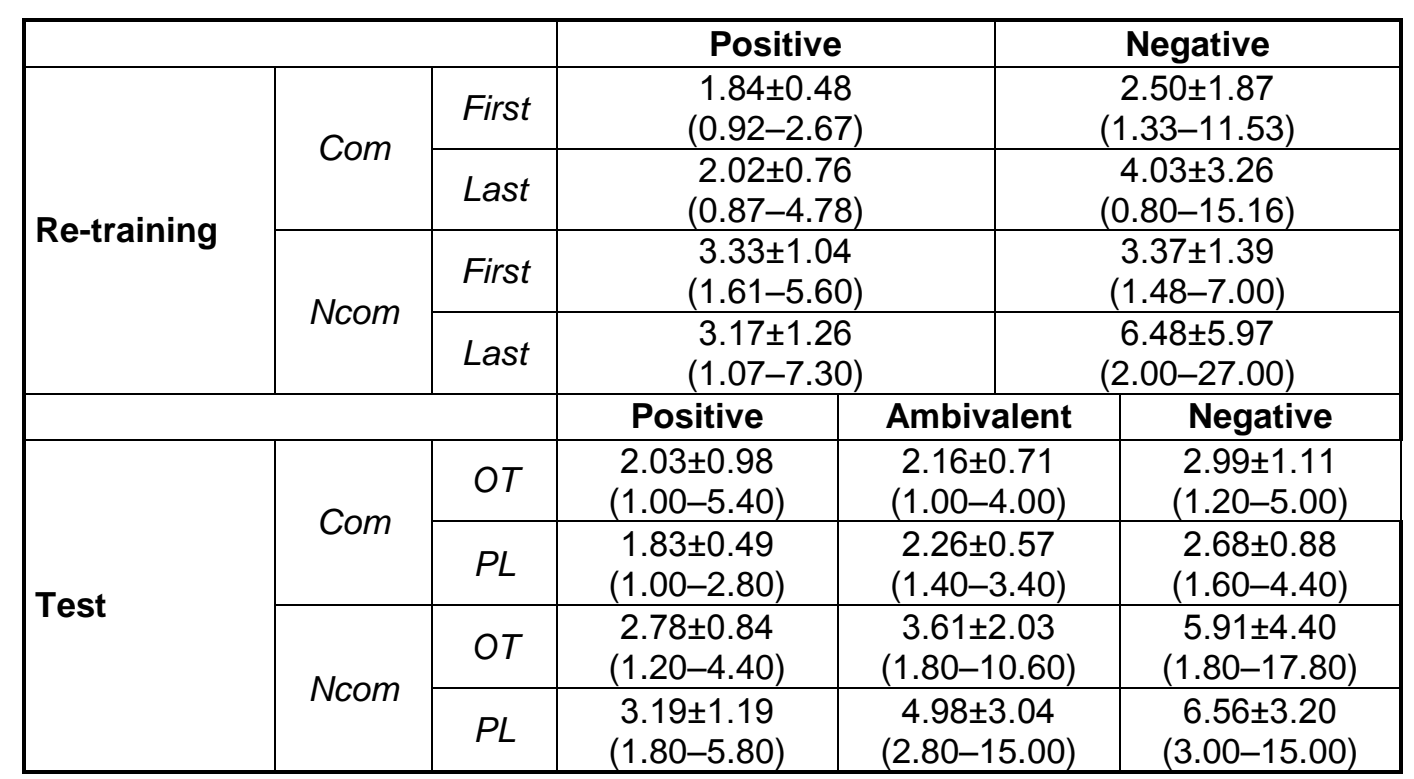

496 